Article

\title{
Gastric Volume Changes in Preterm Neonates during Intermittent and Continuous Feeding-GRV and Feeding Mode in Preterm Neonates
}

\author{
Rozeta Sokou 1,*(D), Ioanna N. Grivea ${ }^{2}$, Eleni Gounari ${ }^{3}$, Polytimi Panagiotounakou ${ }^{1}$, Maria Baltogianni ${ }^{4}$, \\ George Antonogeorgos ${ }^{1}$, Fedra Kokori ${ }^{5}$, Aikaterini Konstantinidi ${ }^{1}{ }^{(D)}$ and Antonios K. Gounaris ${ }^{2}$
}

1 Neonatal Clinic-NICU, General Hospital of Nikaia "Agios Panteleimon", 3 D. Mantouvalou Str., 18454 Piraeus, Greece; ppppolytimi04@gmail.com (P.P.); gantonogeorgos@gmail.com (G.A.); kmaronia@gmail.com (A.K.)

2 Neonatal Clinic-NICU, University General Hospital of Larissa, 41222 Larissa, Greece; ioanna.grivea@gmail.com (I.N.G.); angounaris@med.uth.gr (A.K.G.)

3 Royal Alexandra Children's Hospital Brighton, Eastern Road, Brighton BN2 5BE, UK; elenigounari@gmail.com

4 Neonatal Clinic-NICU, University General Hospital of Ioannina, 45110 Ioannina, Greece; mbalt@doctors.org.uk

5 Department of Radiology, General Hospital “Agios Panteleimon”, 18454 Piraeus, Greece; gkandilis@yahoo.gr

* Correspondence: sokourozeta@yahoo.gr; Tel.: +30-6945107498

Citation: Sokou, R.; Grivea, I.N.; Gounari, E.; Panagiotounakou, P.; Baltogianni, M.; Antonogeorgos, G.; Kokori, F.; Konstantinidi, A.; Gounaris, A.K. Gastric Volume Changes in Preterm Neonates during Intermittent and Continuous Feeding-GRV and Feeding Mode in Preterm Neonates. Children 2021, 8, 300. https://doi.org/10.3390/ children 8040300

Academic Editor: Elizabeth Asztalos

Received: 5 March 2021

Accepted: 13 April 2021

Published: 15 April 2021

Publisher's Note: MDPI stays neutral with regard to jurisdictional claims in published maps and institutional affiliations.

Copyright: (c) 2021 by the authors. Licensee MDPI, Basel, Switzerland. This article is an open access article distributed under the terms and conditions of the Creative Commons Attribution (CC BY) license (https:// creativecommons.org/licenses/by/ $4.0 /$ )
Abstract: Background: We aimed to evaluate gastric volume changes during intermittent milk feeds (IMF) and continuous milk feeds (CMF) in very premature neonates (VPN), with gastric residual volume (GRV) based on antral cross-sectional area (ACSA) measurements and to examine if there were differences in GRV between the two feeding methods. Methods: A randomized prospective clinical trial with crossover design was conducted in 31 preterm neonates (gestational age $<30$ weeks). Gastric volume was assessed twice in each neonate (during IMF and CMF feeding), at 7 specific time points during a 2-h observation period by measuring ACSA changes via the ultrasound (U/S) method. Results: There was a significantly different pattern of gastric volume changes between the two feeding methods. GRV, expressed as the median percentage of ACSA measurement at 120 min relative to the higher ACSA measurement during IMF, was found to be 3\% (range 0-25\%) for IMF and 50\% (range 15-80\%) for CMF. Neonates fed with IMF had a shorter mean gastric emptying time compared to those fed with CMF ( $p=0.0032)$. No signs of feeding intolerance were recorded in either group during the period of observation. Conclusions: Our results showed that gastric volume changes and gastric emptying time in VPN, based on ACSA measurement changes, depend on the milk feeding method. No gastrointestinal complications/adverse events were noted with GRV up to $80 \%$ with CMF.

Keywords: continuous feeding; feeding intolerance; gastric emptying time; gastric residual volume; intermittent feeding; very low birth weight newborns

\section{Introduction}

Feeding intolerance, defined as the inefficiency of the gastrointestinal system of very preterm neonates (VPN) to digest milk, is associated with the presence of increased prefeed gastric residual volumes (GRVs) and expressed with vomiting/abdominal distention/desaturations/bradycardia/apnea, often leading to the disruption of a feeding plan in this fragile population.

During the first weeks of life, very low birth weight (VLBW) and especially extremely low birth weight (ELBW) neonates, commonly exhibit feeding intolerance and a delay in gastric emptying [1-3]. Gastric emptying can act as an indicator of feeding tolerance and it is assessed by measuring the quantity and the quality of aspirated GRV. Gastric emptying 
time in VPN depends on the volume and type of milk and is independent of postnatal age [4]. Several factors such as milk type (breast milk or formula), use of breast milk fortifier, infusion rate, body position and feeding mode (intermitted-bolus or continuous) influence gastric emptying in preterm neonates [5,6]. The most appropriate feeding method (intermittent or continuous) for preterm neonates to achieve full enteral feeding remains an issue of controversy.

A meta-analysis conducted by Premji et al. [7] showcased the difficulties in making universal recommendations regarding the best feeding method for preterm infants with a birth weight less than $1500 \mathrm{~g}$.

There is currently ongoing research on increased GRV in intermittently fed preterm neonates and the incidence of necrotizing enterocolitis (NEC). There are controversies regarding the correlation between increased GRV and NEC in preterm neonates, as the relevant data are insufficient [8-12]. Several methods of assessing the GRV have been trialed in preterm neonates such as the gastric aspiration technique via gastric tube and imaging techniques, but wide variations exist as to what constitutes significant GRV. The ultrasound (U/S) method has been used successfully to investigate gastric emptying in preterm infants [13]. Perrella SL et al. [14] showed that there was a significant relation between antral cross-sectional area (ACSA) and the proportion of feed volume delivered $(p<0.001)$. Furthermore, this method presents a more physiological and non-invasive technique [15].

Limited data are available from trials which have compared bolus versus continuous feeding and the existing evidence is insufficient to determine an optimal feeding strategy. Yan Wang et al. [16], in a meta-analysis of randomized controlled trials have found that intermittent feeding may be more beneficial for low birth weight infants, but well-designed studies and evidenced-based clinical practice are required to determine the most appropriate feeding method for premature infants with low birth weight.

Although in two different publications $[17,18]$ the same upper threshold levels of GRV were applied for determining feeding intolerance during both continuous and intermittent methods of feeding, there are still controversies regarding what constitutes "normal GRV" in VPN fed by continuous feeding.

The aim of our study was to record and evaluate gastric volume changes during intermittent and continuous method of feeding in VPN and to assess GRV based on ACSA measurements, as well as to examine if there are differences in GRV between the two feeding methods. Our secondary aim was to compare gastric emptying time between intermittent and continuous feeding mode, as well as gastrointestinal complications of VPN fed with the two feeding methods.

\section{Materials and Methods}

This single-center, randomized, prospective crossover clinical trial was conducted at the Neonatal Intensive Care Unit (NICU) of Agios Panteleimon Hospital, over a period of one year. The Institutional Review Board approved the protocol (18 May 2016, 29/2), which was designed, conducted, and reported in compliance with the Declaration of Helsinki. Parental informed consent was obtained prior to recruitment.

\subsection{Participants}

The study population consisted of preterm neonates with a gestational age $(G A)<30$ weeks, hospitalized in the NICU. Exclusion criteria included severe congenital abnormalities, ventilator dependence beyond the first week of life, as well as stage II or III NEC according to the modified Bell criteria [19]. Recruitment procedure data are presented in the flow chart (Figure 1). 


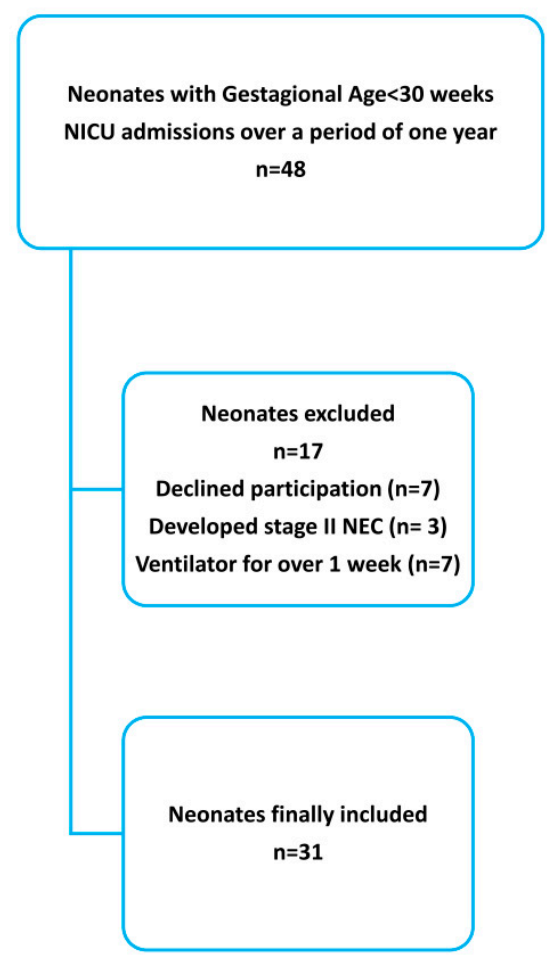

Figure 1. Flow chart of study population.

\subsection{Study Protocol}

The 31 newborn babies included in the study were randomly assigned to be fed with one of the two feeding methods, either intermittent-bolus (group A) or continuous (group B) and underwent serial ultrasound measuring of ACSA changes during a 2-h observation period. Sample size calculation was performed prior to study design in order to compute the sufficient study sample size. The study was powered at $80 \%$ to detect significant standardized effect size differences of 0.25 between two factors in repeated measurements of analysis of variance, under the assumption of a correlation coefficient between measurements $r=0.5$ at a significance level $a=0.05$.

During continuous feeding, neonates received the daily milk volume by orogastric tube (OGT) with a constant rate for $24 \mathrm{~h}$ via a pump. During intermittent-bolus feeding, neonates received OGT feeds via gravity for less than 10 min every $2 \mathrm{~h}$. The pre-feed assessments of GRV were performed through aspiration before every second feed by the responsible nurse. In our NICU, VPN were comfortably contained within the nests in the supine, prone or right lateral position interchangeably. Neonates were fed in the supine position.

All neonates were enrolled in the study within the first week of life. Randomization for the crossover design was achieved with the use of sealed opaque envelopes. The simplest model $\mathrm{AB} / \mathrm{BA}$ was used for randomization. Subjects allocated to the AB study arm received intervention $A$ first, followed by intervention $B$, and vice versa in the $B A$ arm. All study neonates received both modes of feeding and they acted as controls of themselves. The observation period started when the neonates were receiving enteral milk volume of more than $80 \%$ of their daily fluid intake. Sixteen neonates were initially assigned to the bolus feeding group and subsequently to the continuous feeding group, while the remaining 15 were assigned vice versa. Before each measurement, the neonates had been on the same feeding regimen (type and milk volume) for at least $48 \mathrm{~h}$. In all cases, there was a minimum of three days washout interval between the two measurements to eliminate any carry-over effects, but sometimes this period was extended up to five days depending on the availability of the radiologists 
According to the NICU protocol, in VPN enteral feeding was initiated on the 1st-2nd day of life, depending on their clinical status. Minimal enteral feeding was initially introduced at $1 \mathrm{~mL}$ and up to $2 \mathrm{~mL}$ every $2 \mathrm{~h}$ for about three days, depending on the neonate's level of care. Bolus feeding through OGT via gravity was the standard method of feeding in our NICU. The responsible nurse placed the OGT after taking a measurement of the distance from the nose to the ear and the end of the sternum, with the addition of $1 \mathrm{~cm}$ and the correct placement was checked with the aspiration of gastric content. X-rays obtained for other clinical reasons helped with the final assessment of gastric tube placement. Following minimal feeding, standard milk increments of about $20-30 \mathrm{~mL} / \mathrm{kg} /$ day were administered every $2 \mathrm{~h}$ until full enteral feeding was reached (at a volume of $200-210 \mathrm{~mL} / \mathrm{kg} /$ day) in an effort to achieve a weight gain of $>15-20 \mathrm{~g} /$ day.

Every neonate received the same type of milk (breast milk with fortifier or a preterm formula containing $81 \mathrm{kcal} / 100 \mathrm{~mL}$ ) during the 48 -h period before each measurement. The median milk quantity administered to each newborn during the procedure was $17 \mathrm{~mL} / 2 \mathrm{~h}$ (range 9-22) and this amount remained the same in both measurements.

It is worth noting that each newborn was on the same respiratory support during both assessments; either on bubble nasal continuous positive airway pressure (nCPAP) with pressure up to $7 \mathrm{~cm} \mathrm{H}_{2} \mathrm{O}$, or oxygen via incubator with $\mathrm{FiO}_{2}<30 \%$, or room air. During the study period, no neonate received caffeine and nCPAP was used for apnea treatment. Signs of feeding intolerance and any gastrointestinal complications presenting during the observation period which included the day of each measurement and a 48-h period before that, were recorded in detail by the nursing staff caring for each neonate.

\subsection{Ultrasound Examination}

The ultrasound method proposed by Newell et al. [20], was used to evaluate gastric volume changes. After the 10 a.m. morning feed, a radiologist tracked and measured the changes of ACSA using U/S equipment. Measurements were obtained over the next $2 \mathrm{~h}$; every $10 \mathrm{~min}$ for the first $30 \mathrm{~min}$ and thereafter every $30 \mathrm{~min}$; resulting in a total of 7 measurements. Transverse sections of the pyloric antrum were taken, with the vertebral column and origin of the superior mesenteric artery serving as guidelines. The measurements were performed with the newborn lying in a right lateral position. In neonates fed intermittently, gastric residual volume was assessed through aspiration and was thrown away before the measurement procedure was started to ensure that any residual of the previous feeding would not affect the following ACSA measurement. The residual volume was less than $20 \%$ of the previous feeding volume in all of the intermittently fed neonates. The first measurement of the ACSA was conducted immediately after the milk feed administration. In continuously fed neonates, the radiologist measured the ACSA during continuous milk administration via pump, from $10 \mathrm{a} . \mathrm{m}$. and for $2 \mathrm{~h}$ at the same 7 time-specific points.

The ultrasonographic method involves minimal handling; the probe is in touch with the baby's abdomen only for few seconds in every measurement (less than 2 min are needed in total for the 7 measurements) and the whole examination can be performed in the cot. All measurements were taken with the Easote My Lab 50 Ultrasound machine and a 7.5 MHz probe was used. The same radiologist, performed all U/S measurements.

\subsection{Outcome Measures}

ACSA measurements according to Newell's method [20] were recorded at 7 timespecific points for each neonate during the two feeding methods. Gastric content changes were evaluated based on these serial ACSA measurements. GRV was expressed as a percentage of the ACSA measurement at 120 min relative to the higher ACSA measurement during intermittent feeding.

Gastric emptying time was defined as the time needed for the ACSA measurement (in $\mathrm{cm}^{2}$ ) to reach half of its higher value during intermittent feeding for all study neonates (as they were acting as controls of themselves). 
Data on demographics, maternal medication during pregnancy, neonatal physiological parameters, and clinical findings were recorded. Small for gestational age (SGA), neonatal respiratory distress syndrome (RDS), NEC and bronchopulmonary dysplasia (BPD, the need for continuous supplemental oxygen at 28 days of age), defined according to the literature $[19,21-23]$, were also recorded. Regurgitation, vomiting, bile or blood stained aspirates, visible bowel loops, abdominal distension and heme-positive stools, were considered as possible gastrointestinal complications. In neonates fed intermittently, gastric residue of more than $50 \%$ of the previous feeding volume was also considered as a possible gastrointestinal complication.

\subsection{Statistical Analysis}

The normality of the distribution of the variables was evaluated using statistical tests and diagnostic graphs (Shapiro-Wilks and Kolmogorrov-Smironv tests of normality and P-P plots and Q-Q plots, accordingly). Continuous variables are presented as mean and standard deviation if they are normally distributed or median and 1st and 3rd quartile if their distribution is skewed. Categorical variables are presented as absolute and relative frequencies. The Wilcoxon matched paired rank test was used in order to assess differences in ACSA between bolus and continuous fed VLBW infants at each time point. Repeated measured analysis of variance (repeated ANOVA) was applied, after a log-transformation of ACSA values for complying with the assumption of normality, in order to assess the effect of the feeding method (bolus vs. continuous) in the changes of ACSA over time, adjusting for the possible confounding effect of gender, ventilation status (nCPAP) and birth weight in all the different time points. In order to address the $p$-value inflation due to multiple statistical comparisons, the Bonferroni correction was applied. All reported probability values ( $p$-values) are based on two sided tests and compared to a significance level of 5\%. SPSS 18.0 software was used for the all calculations (SPSS Inc. Released 2009. PASW Statistics for Windows, Version 18.0. SPSS Inc., Chicago, IL, USA).

\section{Results}

The clinical characteristics of the study population are presented in Table 1.

Table 1. Baseline characteristics of the neonates study.

\begin{tabular}{cc}
\hline Number of neonates included & 31 \\
Males /Females & $14 / 17$ \\
BW g (mean, SD) & $982(28)$ \\
GA weeks (mean, SD) & $28(1.5)$ \\
SGA (n) & $7 / 31$ \\
Antepartum corticosteroids & $19 / 31$ \\
1 min Apgar Score (median, range) & $6(1-9)$ \\
5 min Apgar Score (median, range) & $8(2-9)$ \\
Inborns / outborns (n) & $15 / 16$ \\
RDS (n) & 29 \\
BPD (n) & 19 \\
nCPAP & \\
O 2 30\% & $18(58.06)$ \\
room air & $7(22.58)$ \\
Respiratory support during measurements (n, \%) & $6(19.35)$ \\
Full enteral feeding days (median, range) & $12(10-21)$ \\
First measurement (days/median, range) & $15.5(11-32)$ \\
Second measurement (days/median, range) & $19(14-35)$ \\
Breast milk + suppl/formula (n) & $17(9-22)$ \\
Milk volume (mL/2 h) during the assessments (median, range) & $21 / 10$ \\
\hline Abbreviations: BPD, bronchopulmonary dysplasia; BW, birth weight; GA, gestational age; nCPAP, nasal continu- \\
ous positive airway pressure; RDS, respiratory distress syndrome.
\end{tabular}


The first measurements were performed on the 15th (median, range 11-32) day of life and the second ones on the 19th (median, range 14-35) day of life.

The mean log-transformed ACSA values for continuously fed newborns were significantly lower at 0,10,20 and $30 \mathrm{~min}(p<0.001)$ and significantly higher at 90 and $120 \mathrm{~min}$ $(p<0.001)$ compared to the respective ACSA values for intermittently fed neonates. At 60 min the ACSA values were not significantly different between the two groups. (Table 2, Figure 2).

Table 2. Distribution of ACSA values (median/1st-3rd Quartile) according to feeding method (bolus vs. continuous) in the ultrasound (U/S) assessment time points with the corresponding $p$ value.

\begin{tabular}{cccc}
\hline & \multicolumn{2}{c}{ ACSA $\mathbf{( c m}^{\mathbf{2}} \mathbf{l}$} & \\
\hline $\begin{array}{c}\text { Assessment Time after the End of } \\
\text { Milk Administration }\end{array}$ & Bolus Feeding & Continuous Feeding & $p$-Value \\
\hline $0^{\prime}$ & $1.9(1.4-3.6)$ & $1.0(0.6-1.7)$ & $<0.001$ \\
$10^{\prime}$ & $1.8(1.5-3.0)$ & $1.0(0.7-1.7)$ & $<0.001$ \\
$20^{\prime}$ & $1.5(1.2-2.5)$ & $0.9(0.6-1.7)$ & $<0.001$ \\
$30^{\prime}$ & $1.3(1.0-2.0)$ & $0.8(0.5-1.8)$ & $<0.001$ \\
$60^{\prime}$ & $1.0(0.5-1.0)$ & $0.8(0.5-1.8)$ & 0.328 \\
$90^{\prime}$ & $0.4(0.2-0.6)$ & $0.7(0.4-1.4)$ & $<0.001$ \\
$120^{\prime}$ & $0.1(0.0-0.2)$ & $0.8(0.5-1.95)$ & $<0.001$ \\
\hline
\end{tabular}

Abbreviations: on antral cross-sectional area (ACSA).

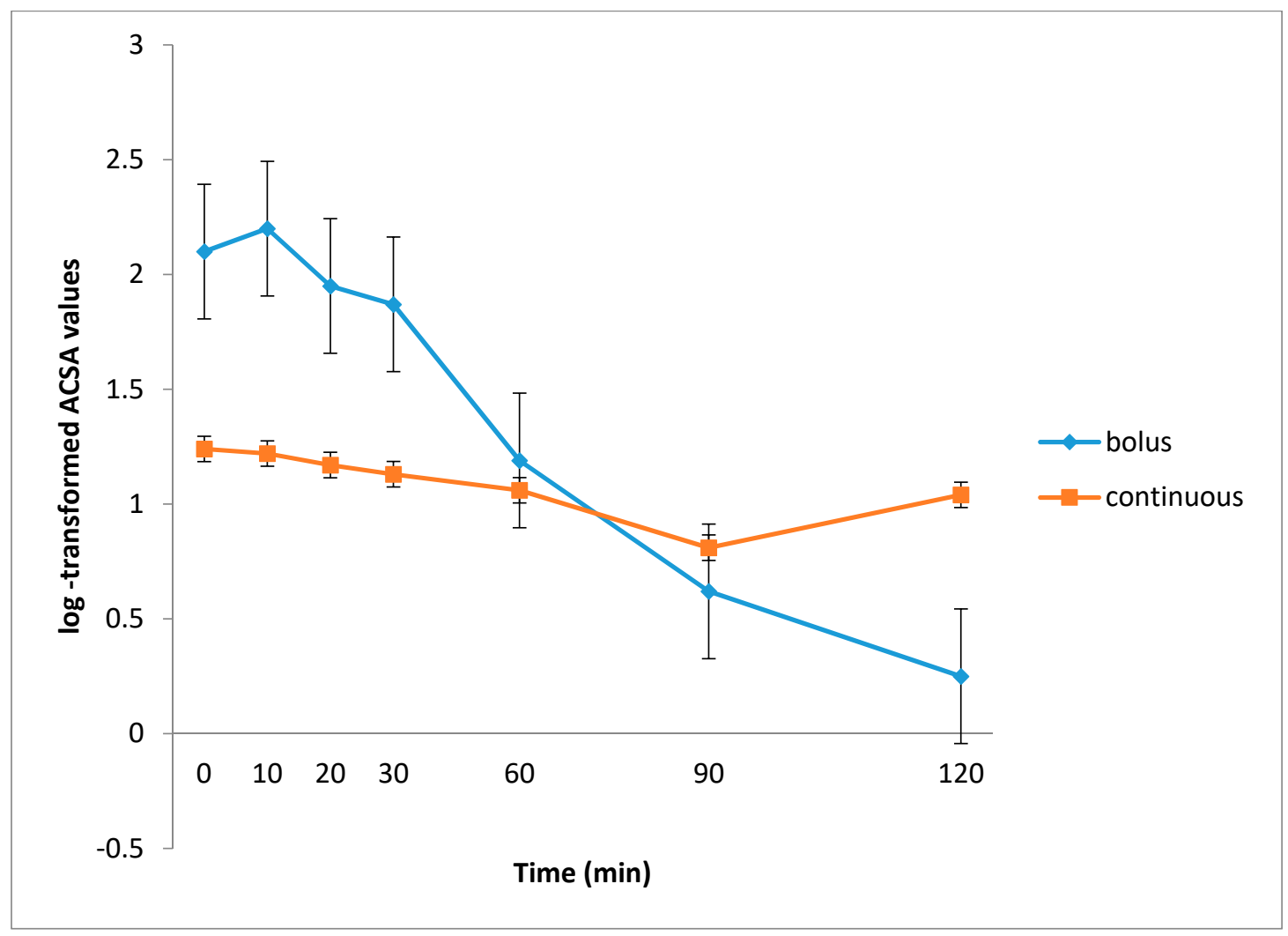

Figure 2. Pattern of gastric antral cross-sectional area (ACSA) values (log-transformed) reduction through different study time points according to feeding method (intermittent vs. continuous).

Error bars represent standard error of the mean.

Moreover, the variability in ACSA values at each time point, expressed as an interquartile range (3rd quartile value minus 1st quartile) were assessed and found to be very low during the continuous feeding method (interquartile range varying from 1.0 to 
$1.45 \mathrm{~cm}^{2}$ ) while during the intermittent feeding method they were very high (interquartile range varying from 0.2 to $2.2 \mathrm{~cm}^{2}$ ). (Table 2). In the U/S measurement at 120 min median ACSA values were $0.1 \mathrm{~cm}^{2}$ and $0.8 \mathrm{~cm}^{2}$ for intermittently and continuously fed neonates, respectively, and this difference was statistically highly significant $(p<0.001)$.

GRV, expressed as the percentage of the last ACSA measurement (at $120 \mathrm{~min}$ ) relative to the higher ACSA level, was found to be $50 \%$ (median, range $15-80 \%$ ) during continuous feeding. It is noteworthy that although 13 out of the 31 neonates had GRV values between $50-80 \%$ with continuous feeding, no gastrointestinal complications were recorded. For intermittent feeding, GRV value was 3\% (median, range 0-25\%).

There was a statistically significant difference in the mean gastric emptying time between the intermittently fed (group A) and continuously fed (group B) neonates [37.8 \pm 15 min vs. $57.7 \pm 29 \mathrm{~min}(p=0.0032)]$. For neonates fed continuously, gastric emptying time was not calculated in all cases, as 11 out of 31 neonates' ACSA measurement did not reach half of the higher value.

During the observation period, no infant displayed signs of feeding intolerance such as vomiting, apnea, bile/blood stained aspirate, visible bowel loops, abdominal distension and no infant developed NEC.

\section{Discussion}

This single-center, randomized, prospective crossover clinical trial showed that in very preterm neonates, the milk feeding method had an influence on gastric volume changes as well as on gastric emptying time, based on ACSA measurement changes. Moreover, no gastrointestinal complications/adverse events were noted when GRV was up to $80 \%$ in continuously fed VPN.

There is an ongoing debate among neonatologists throughout the years regarding the optimal timing, quantity, and method for feeding VLBW neonates. One meta-analysis concluded that developing universal recommendations regarding the best feeding method for infants less than $1500 \mathrm{gr}$ is problematic [7] and more recently Wang et al. [16] reported that intermittent feeding may be more beneficial for low birth weight neonates, but welldesigned and evidenced-based studies are required to determine the most appropriate feeding method for preterm neonates.

Continuous enteral feeding has been shown to help limit the risk of hypoxic-ischemic gut damage in critically ill preterm neonates [24]. Continuous feeding also appears to maintain the gastrointestinal hormones, such as gastrin and insulin, at a high level increasing the absorption, reducing energy expenditure [16] and increasing duodenal motility, while bolus feeding decreases it [25]. Dani C. et al. [26], have demonstrated that bolus milk feeding increases splanchnic oxygenation in both appropriate for gestational age (AGA) and SGA infants, whereas continuous feeding does not.

Extensive research has been conducted by neonatologists on what exactly constitutes an "acceptable" or "normal" level of GRV following an intermittent feeding regime [27-29] but controversies persist as to what constitutes a "normal" GRV during continuous feeding, which would permit the uneventful continuation of enteral feeding of preterm neonates. Dollberg S. et al. [17], considering the same GRV upper threshold of $<40 \%$ as acceptable for both intermittent and continuous feeding methods, came to the conclusion that the intermittent feeding method is more effective than continuous in improving feeding tolerance in small VLBW infants. Dsilna A. et al. [18] also adopted similar upper thresholds $(<50 \%)$ as indicators of feeding tolerance for these two feeding methods, and found that continuous feeding seems to be better than intermittent feeding with regards to gastrointestinal tolerance and growth of very preterm neonates. On the other hand, Rovekamp-Abels LW et al. [30], in a recent study on bolus and semi-continuous feeding methods in very preterm neonates, evaluated using a larger gastric residue prior to postponing feeding and concluded that bolus and continuous feeding are equally suitable strategies for these neonates. 
Despite the wide variation regarding the definition of an abnormal GRV, the most cited GRV considered as abnormal is the one that exceeds the hourly infusion rate or $50 \%$ of milk administered every two hours [12]. In our study upper GRV thresholds during bolus feeding were similar to those already reported. For the first time in the literature, we found GRV up to $80 \%$ to be well tolerated during continuous feeding. Thus, our results could lead to the consideration of a higher upper threshold for GRV during continuous feeding and might help re-evaluate the current knowledge base.

The usefulness of the systematic evaluation of GRV in VPN is the subject of a longstanding debate among neonatologists. Some authors propose omitting the routine evaluation of pre-feed GRV during bolus feeding [12,31] and more recently others [32], suggest that the omission of gastric residual evaluation in VPN should translate into evidencebased practice, as it increases the delivery of enteral nutrition and improves weight gain leading to earlier hospital discharge. On the other hand, a recent meta-analysis came to the conclusion that there is low quality evidence to suggest that routine monitoring of GRV increases the risk of feed interruption episodes as well as the time to reach full enteral feeding [10].

High GRV is a sign of feeding intolerance and has been used as an early marker to prevent NEC. Both Cobb B.A. et al. [8] and Bertino E. et al. [9] found a correlation between high GRV and NEC. The aforementioned meta-analysis [10] concluded that there were insufficient data to support or not GRV evaluation for the prevention of NEC. On the contrary, Parker L.A. et al. [32] supported the omission of gastric residual evaluation in extremely preterm neonates, although the study was not directed to evaluate the risk of NEC In this study, abdominal distention. as well as. increasing abdominal girth $(>2 \mathrm{~cm})$ were used as signs of feeding intolerance. In relation to the above we must take into account that during nCPAP support, extremely preterm neonates present with benign abdominal distention ("CPAP belly syndrome") in a percentage ranging from 45\% [33] up to 90\% [34] and in this case, abdominal distention is not a reliable indicator of feeding intolerance.

$\mathrm{U} / \mathrm{S}$ is a useful method for the evaluation of gastric emptying in different clinical situations in preterm infants [35]. In the present study, the use of U/S allowed us to monitor milk clearance from the stomach over a 2-h period during both intermittent and continuous feeding methods. It is clear that ACSA measurements in intermittent-bolus feeding were significantly higher in the first $30 \mathrm{~min}$, a difference that no longer existed at $60 \mathrm{~min}$, and was subsequently reversed, with ACSA measurements becoming significantly higher in the continuously fed group at the 90 min and especially 120 min mark.

There are limitations in the current study. The most important limitation is the small sample size. Another limitation is that the gastric emptying time in continuously fed neonates was not successfully measured in all cases. Nonetheless, the strength of this study derives from the fact that the newborns were acting as controls of themselves and were fed with the same quantity and type of milk during both intervention periods (bolus and continuous feeding). Moreover, for each newborn, gastric volume changes based on ACSA measurements were serially evaluated during a 2-h period of $U / S$ assessment.

\section{Conclusions}

In very preterm neonates, gastric emptying seems to depend on the mode of feeding. Our study showed that in neonates fed with the continuous feeding mode, no gastrointestinal complications were noted despite the fact that gastric residual volume based on ACSA values calculated reached up to $80 \%$ of gastric feeding volume. Thus, during continuous feeding, gastric residual volumes up to $80 \%$ could be acceptable. Further studies are necessary to verify our findings, as establishing an acceptable gastric residual in continuous feeding may justify changes in nutritional practices for this very susceptible population. 
Author Contributions: Conceptualization: A.K.G. and R.S.; methodology: A.K.G., R.S., and I.N.G.; software: A.K.G. and R.S.; validation A.K., R.S., P.P. and A.K.G.; formal analysis: G.A. and A.K.G.; data curation: R.S., P.P., A.K., F.K., E.G. and A.K.G.; writing-original draft preparation: R.S., E.G., I.N.G., M.B. and A.K.G.; writing—review and editing: A.K.G., R.S. and E.G.; project administration: A.K.G. All authors have read and agreed to the published version of the manuscript.

Funding: This research did not receive any specific grant from funding agencies in the public, commercial, or not-for-profit sectors.

Institutional Review Board Statement: The study was conducted according to the guidelines of the Declaration of Helsinki, and approved by the Institutional Review Board of of Nikaia General Hospital “Agios Panteleimon" (29/2, 18 May 2016).

Informed Consent Statement: Parental informed consent was obtained for all neonates involved in the study.

Data Availability Statement: The data presented in this study are available on request from the corresponding author.

Acknowledgments: Authors would like to thank Christos Costalos for his valuable contribution to all parts of the study.

Conflicts of Interest: The authors declare that they have no conflict of interest.

\section{References}

1. Herbst, J.J. Development of suck and swallow. J. Pediatr. Gastroenterol. Nutr. 1983, 2 (Suppl. 1), S131-S135. [CrossRef]

2. Davidson, G. The Role of Lower Esophageal Sphincter Function and Dysmotility in Gastroesophageal Reflux in Premature Infants and in the First Year of Life. J. Pediatr. Gastroenterol. Nutr. 2003, 37, S17-S22. [CrossRef]

3. Berseth, C.L. Gastrointestinal motility in the neonate. Clin. Perinatol. 1996, 23, 179-190. [CrossRef]

4. Ferreira, C.H.F.; Martinez, F.E.; Crott, G.C.; Belik, J. Gavage Feed Volume Determines the Gastric Emptying Rate in Preterm In-fants. J. Pediatr. Gastroenterol. Nutr. 2018, 67, e43-e46. [CrossRef] [PubMed]

5. Perrella, S.L.; Hepworth, A.R.; Simmer, K.N.; Geddes, D.T. Influences of Breast Milk Composition on Gastric Emptying in Preterm Infants. J. Pediatr. Gastroenterol. Nutr. 2015, 60, 264-271. [CrossRef] [PubMed]

6. Cohen, S.; Mandel, D.; Mimouni, F.B.; Solovkin, L.; Dollberg, S. Gastric residual in growing preterm infants: Effect of body posi-tion. Am. J. Perinatol. 2004, 21, 163-166. [CrossRef] [PubMed]

7. Premji, S.S.; Chessell, L. Continuous nasogastric milk feeding versus intermittent bolus milk feeding for premature infants less than 1500 grams. Cochrane Database Syst. Rev. 2011, 2011, CD001819. [CrossRef] [PubMed]

8. Cobb, B.A.; Carlo, W.A.; Ambalavanan, N. Gastric residuals and their relationship to necrotizing enterocolitis in very low birth weight infants. Pediatrics 2003, 113 Pt 1, 50-53. [CrossRef]

9. Bertino, E.; Giuliani, F.; Prandi, G.; Coscia, A.; Martano, C.; Fabris, C. Necrotizing Enterocolitis: Risk Factor Analysis and Role of Gastric Residuals in Very Low Birth Weight Infants. J. Pediatr. Gastroenterol. Nutr. 2009, 48, 437-442. [CrossRef]

10. Abiramalatha, T.; Thanigainathan, S.; Ninan, B. Routine monitoring of gastric residual for prevention of necrotising entero-colitis in preterm infants. Cochrane Database Syst. Rev. 2018, 2018, CD012937.

11. Ou, J.; Courtney, C.M.; Steinberger, A.E.; Tecos, M.E.; Warner, B.W. Nutrition in Necrotizing Enterocolitis and Following Intesti-nal Resection. Nutrients 2020, 12, 520. [CrossRef] [PubMed]

12. Parker, L.; Torrazza, R.M.; Li, Y.; Talaga, E.; Shuster, J.; Neu, J. Aspiration and evaluation of gastric residuals in the neonatal in-tensive care unit: State of the science. J. Perinat. Neonatal Nurs. 2015, 29, 51. [CrossRef] [PubMed]

13. Gounaris, A.K.; Grivea, I.N.; Baltogianni, M.; Gounari, E.; Antonogeorgos, G.; Kokori, F.; Panagiotounakou, P.; Theodoraki, M.; Konstantinidi, A.; Sokou, R. Caffeine and Gastric Emptying Time in Very Preterm Neonates. J. Clin. Med. 2020, 9, 1676. [CrossRef] [PubMed]

14. Perrella, S.L.; Hepworth, A.R.; Simmer, K.N.; Geddes, D.T. Validation of Ultrasound Methods to Monitor Gastric Volume Changes in Preterm Infants. J. Pediatr. Gastroenterol. Nutr. 2013, 57, 741-749. [CrossRef]

15. Perrella, S.L.; Hepworth, A.R.; Simmer, K.N.; Hartmann, P.E.; Geddes, D.T. Repeatability of Gastric Volume Measurements and Intragastric Content Using Ultrasound in Preterm Infants. J. Pediatr. Gastroenterol. Nutr. 2014, 59, 254-263. [CrossRef]

16. Wang, Y.; Zhu, W.; Luo, B.-R. Continuous feeding versus intermittent bolus feeding for premature infants with low birth weight: A meta-analysis of randomized controlled trials. Eur. J. Clin. Nutr. 2019, 74, 775-783. [CrossRef] [PubMed]

17. Dollberg, S.; Kuint, J.; Mazkereth, R.; Mimouni, F.B. Feeding tolerance in preterm infants: Randomized trial of bolus and con-tinuous feeding. J. Am. Coll. Nutr. 2000, 19, 797-800. [CrossRef]

18. Dsilna, A.; Christensson, K.; Alfredsson, L.; Lagercrantz, H.; Blennow, M. Continuous feeding promotes gastrointestinal toler-ance and growth in very low birth weight infants. J. Pediatr. 2005, 147, 43-49. [CrossRef]

19. Walsh, M.C.; Kliegman, R.M. Necrotizing Enterocolitis: Treatment Based on Staging Criteria. Pediatr. Clin. N. Am. 1986, 33, 179-201. [CrossRef] 
20. Newell, S.J.; Chapman, S.; Booth, I.W. Ultrasonic assessment of gastric emptying in the preterm infant. Arch. Dis. Child. 1993, 69, 32-36. [CrossRef]

21. Walti, H.; Couchard, M.; Relier, J.P. Neonatal diagnosis of respiratory distress syndrome. Eur. Respir. J. Suppl. 1989, 3, 22s-26s.

22. Schlaudecker, E.P.; Munoz, F.M.; Bardají, A.; Boghossian, N.S.; Khalil, A.; Mousa, H.; Nesin, M.; Nisar, M.I.; Pool, V.; Spiegel, H.M.; et al. Small for gestational age: Case definition \& guidelines for data collection, analysis, and presentation of maternal immunisation safety data. Vaccine 2017, 35, 6518-6528. [CrossRef] [PubMed]

23. Jobe, A.H.; Bancalari, E. Bronchopulmonary Dysplasia. Am. J. Respir. Crit. Care Med. 2001, 163, 1723-1729. [CrossRef] [PubMed]

24. Dani, C.; Corsini, I.; Generoso, M.; Gozzini, E.; Bianconi, T.; Pratesi, S. Splanchnic Tissue Oxygenation for Predicting Feeding Tolerance in Preterm Infants. J. Parenter. Enter. Nutr. 2014, 39, 935-940. [CrossRef] [PubMed]

25. Berseth, C.L. Effect of early feeding on maturation of the preterm infant's small intestine. J. Pediatr. 1992, 120, 947-953. [CrossRef]

26. Dani, C.; Pratesi, S.; Barp, J.; Bertini, G.; Gozzini, E.; Mele, L.; Parrini, L. Near-Infrared Spectroscopy Measurements of Splanchnic Tissue Oxygenation During Continuous Versus Intermittent Feeding Method in Preterm Infants. J. Pediatr. Gastroenterol. Nutr. 2013, 56, 652-656. [CrossRef] [PubMed]

27. Bodé, S.; Dreyer, M.; Greisen, G. Gastric Emptying and Small Intestinal Transit Time in Preterm Infants: A Scintigraphic Method. J. Pediatr. Gastroenterol. Nutr. 2004, 39, 378-382. [CrossRef]

28. Enriquez, A.; Bolisetty, S.; Patole, S.; A Garvey, P.; Campbell, P.J. Randomised controlled trial of cisapride in feed intolerance in preterm infants. Arch. Dis. Child. Fetal Neonatal Ed. 1998, 79, F110-F113. [CrossRef]

29. Mihatsch, W.A.; Von Schoenaich, P.; Fahnenstich, H.; Dehne, N.; Ebbecke, H.; Plath, C.; Von Stockhausen, H.-B.; Muche, R.; Franz, A.; Pohlandt, F. The Significance of Gastric Residuals in the Early Enteral Feeding Advancement of Extremely Low Birth Weight Infants. Pediatrics 2002, 109, 457-459. [CrossRef]

30. Rövekamp-Abels, L.W.W.; Hogewind-Schoonenboom, J.E.; de Wijs-Meijler, D.P.; Maduro, M.D.; Jansen-van der Weide, M.C.; van Goudoever, J.B.; Hulst, J.M. Intermittent Bolus or Semicontinuous Feeding for Preterm Infants? J. Pediatr. Gastroenterol. Nutr. 2015, 61, 659-664. [CrossRef]

31. Li, Y.F.; Lin, H.C.; Torrazza, R.M.; Parker, L.; Talaga, E.; Neu, J. Gastric residual evaluation in preterm neonates: A useful monitoring technique or a hindrance? Pediatr. Neonatol. 2014, 55, 335-340. [CrossRef] [PubMed]

32. Parker, L.A.; Weaver, M.; Murgas Torrazza, R.J.; Shuster, J.; Li, N.; Krueger, C.; Neu, J. Effect of Gastric Residual Evaluation on Enter-al Intake in Extremely Preterm Infants: A Randomized Clinical Trial. JAMA Pediatr. 2019, 173, 534-543. [CrossRef] [PubMed]

33. Gounaris, A.; Costalos, C.; Varchalama, L.; Kokori, P.; Kolovou, E.; Alexiou, N. Gastric emptying in very-low-birth-weight in-fants treated with nasal continuous positive airway pressure. J. Pediatr. 2004, 145, 508-510. [CrossRef] [PubMed]

34. Jaile, J.C.; Levin, T.; Wung, J.T.; Abramson, S.J.; Ruzal-Shapiro, C.; Berdon, W.E. Benign gaseous distension of the bowel in prema-ture infants treated with nasal continuous airway pressure: A study of contributing factors. AJR Am. J. Roentgenol. 1992, 158, 125-127. [CrossRef] [PubMed]

35. Beck, C.E.; Witt, L.; Albrecht, L.; Winstroth, A.M.; Lange, M.; Dennhardt, N.; Boethig, D.; Sumpelmann, R. Ultrasound assessment of gastric emptying time in preterm infants: A prospective observational study. Eur. J. Anaesthesiol. 2019, 36, 406-410. [CrossRef] 